\title{
Global mental health in high-income countries
}

S. P. Sashidharan, Ross White, Roberto Mezzina, Stefan Jansen

and Darius Gishoma

\section{Summary}

Over the past decade there have been significant efforts to scale-up mental health services in resource-poor countries. A number of cost-effective innovations have emerged as a result. At the same time, there is increasing concern in resource-rich countries about efficacy, efficiency and acceptability of mental health services. We consider two specific innovations used widely in low- and middle-income countries, task-sharing and a development model of mental healthcare, that we believe have the potential to address some of the current challenges facing mental health services in high-income countries.

\section{Declaration of interest}

S.P.S. and R.M. are involved in international programmes for community mental health supported by the WHO Collaborating Centre, Trieste, Italy where R.M. is the director. S.P.S. is a director of (NGO) Mental Health Rights. R.W. leads the MSc programme in Global Mental Health at the University of Glasgow.

\section{Copyright and usage}

(c) The Royal College of Psychiatrists 2016.
S. P. Sashidharan (pictured) is an honorary professor at the Institute of Health and Wellbeing, University of Glasgow. Ross White is a senior lecturer at the Institute of Health and Wellbeing, University of Glasgow. Roberto Mezzina is Director of the Department of Mental Health and WHO Collaborating Centre for Research and Training in Trieste, Italy. Stefan Jansen is a senior researcher at the Center for Mental Health, College of Medicine and Health Sciences at the University of Rwanda. Darius Gishoma is a senior lecturer at the Center for Mental Health, College of Medicine and Health Sciences at the University of Rwanda.

\section{Learning from global mental health}

Over the past decade, global mental health has emerged as an important area of discourse and research as well as a powerful impetus for mental health service development in low- and middle-income countries (LMIC). Unfortunately, there is a misconception that global mental health is about improving care in just the poorest countries, whereas we believe that global mental health should be about making mental healthcare better everywhere, including high-income countries: global mental health necessitates a truly global focus.

It is argued that wealthy countries, whether they have marketdriven or state-planned systems, have created expensive and inefficient mental healthcare services, and decisions about mental healthcare do not sufficiently involve those who use services and their families. ${ }^{1}$ Although mental healthcare systems vary a great deal in richer counties in most resource-rich countries they remain inaccessible and insensitive $e^{1,2}$ with suggestion of 'widespread evidence of poor quality care' in England, for example. ${ }^{3}$ There is also concern that mental health interventions often fail to make a significant difference to people's lives, especially in relation to their recovery or social inclusion. ${ }^{4}$ This amounts to a 'recovery gap' between what services are prioritising in terms of outcomes, compared with what actually matters to service users and their families. This may be particularly pronounced for marginalised groups such as minority communities in these countries. Further investment in mental health services in high-income countries is unlikely to bring about proportional, qualitative improvements in patient outcome or satisfaction. For this to happen, more fundamental changes in the way mental health services are organised and delivered are necessary. Closer attention will need to be paid to bridging the 'recovery gap' as an integral part of transforming mental healthcare in high-income countries.

We believe that the emerging health capabilities and care methodologies of global mental health, developed and implemented successfully in LMIC, have a role to play here. These may help us address some of the challenges that mental health services currently face in high-income countries and help improve outcomes. This will mean a process of 'reverse transfer' or 'counter-flow' of knowledge and practice from low- and middleincome to high-income countries, based on the experience of mhGAP and related programmes. ${ }^{5}$

Here we consider two specific innovations that have the potential to improve the processes and outcome of mental healthcare systems in high-income countries. These are (a) task-sharing or task-shifting, and (b) a community development model that focuses on livelihood and social inclusion when designing and delivering mental healthcare.

\section{Task-sharing}

Task-sharing (or task-shifting) is defined as delegating tasks to existing or new cadres of workers with either less training or narrowly tailored training. Many healthcare interventions in LMIC are delivered by community workers who are newly recruited and trained for a specific purpose. In mental health, task-sharing helps to achieve a rational redistribution of resources, from specialist mental health professionals, including psychiatrists, psychologists and psychiatric nurses, to nonspecialist health workers in primary care and community settings. In many LMIC, effective and accessible mental healthcare cannot be provided through a system that relies on mental health professionals as they are a scarce and expensive resource. Instead, mental health interventions are delivered by new cadres of community workers, recruited from 'available human resources from the local communities.' ${ }^{6}$ Task-sharing has been successfully employed in delivering complex interventions for several mental health conditions in LMIC. Trials have shown that lay people or community health workers can be trained to deliver effective psychological and psychosocial interventions for people with depressive and anxiety disorders, schizophrenia and dementia in 
a diverse range of LMIC, and task-sharing has been recognised as a key innovation for delivering psychosocial interventions at the World Innovation Summit in Health. ${ }^{5}$ A Cochrane review that summarises the relevant research indicates that task-sharing in mental healthcare in LMIC can improve clinical outcomes for depressive disorders, post-traumatic stress disorder, alcohol use disorders and dementia. ${ }^{7}$

If task-sharing is effective in LMIC, is there any reason to think that this approach would not be equally effective in richer countries? Already in high-income countries, there are models of care that replicate the conventional roles of specialists (psychiatrists and psychiatric nurses) by generalists (primary care) or non-professional, lay workers and peer support. Implementing task-sharing as an integral part of service delivery in mental healthcare in high-income countries offers several advantages. It has the potential to scale down the top heavy, inefficient and seemingly overbureaucratic mental health systems in these countries and will allow the re-allocation of resources to underinvested areas, such as addressing social determinants of mental ill health and services that could enhance well-being, primary prevention, early detection, recovery and psychosocial rehabilitation. Furthermore, we believe that task-sharing, through fostering personalised and humanistic care, has the potential to improve the quality of relationships between patients (especially those with long-term needs and disabilities), their families and service providers. A programme of task-sharing, delivering routine and complex mental healthcare interventions based on the experience from LMIC, would also tackle the potential shortage in the health and social care workforce. One of the biggest challenges for today's professional workforce is that it was trained and developed to work within a model centred around single episodes of treatment in hospital, whereas those placing the greatest demand on services are likely to be those who need integrated, long-term health and social care. ${ }^{8}$ This is highly relevant when providing sustained, humanistic and personcentred care and support for people with complex and long-term mental health problems in the community.

To be successful in high-income country settings, task-sharing requires a health systems approach that includes ongoing training and professional development, supportive supervision, clear referral pathways to specialist care and a clear role for the non-specialist within the health system. ${ }^{6}$ Factors such as remuneration and training, are also important. The necessary infrastructure for developing task-sharing is clearly available in most high-income countries. For example, training and supervision of new cadres of staff should be possible within well-developed mental health teams. Care methodologies, such as a case management and care programme approach will underpin task-sharing and ensure integrated care. As Patel points out, ${ }^{6}$ mental health is too important to be left to mental health professionals alone and all communities are richly endowed with people who are capable of caring for those with mental health problems.

\section{Development approach in mental healthcare}

A major fault line in the way mental healthcare is organised in many high-income countries is the separation of healthcare (services largely confined to detection and treatment of mental disorders) from social care (addressing the social determinants of poor health and the environmental context). This leads to an imbalance emerging between the priority given to a biomedical approach and the relative lack of resources for addressing the broader social determinants of mental ill health. Mental healthcare tends to be dominated by the views of healthcare professionals and focuses on specific disorders or conditions. As a result, priorities in mental healthcare remain narrowly defined with disproportionate investment in a biomedical approach.

An important innovation in global mental health is the 'bottom up' or grass-roots approach to developing and designing mental health services. This approach serves to increase the uptake of services, while also improving social outcomes for service users and at the community level..$^{9}$ Such changes are unlikely to be achieved if mental health services remain remote from the local communities. A professionally driven or 'top down' approach to developing services is unlikely to address the social and material determinants of mental ill health and its outcome. This requires the adoption of a development model for planning, commissioning and delivering care.

The experience of mainly non-governmental organisations (NGOs) working in resource-poor settings shows the importance of adopting a development model when planning and implementing mental healthcare. The development approach involves mobilising, training and sensitising relevant mental health and development stakeholders in any given community. Although appropriate medical interventions are delivered through community mental health programmes, there is an equal emphasis on helping people with mental health problems to gain or regain the ability to work, to earn and to contribute to their family and community. ${ }^{9}$ For example, the work of Basic Needs has reached over half a million people with mental health problems, their carers and family members in some of the poorest parts of the world. ${ }^{10}$ This approach has resulted in a significant increase in those accessing treatment and, at the same time, appears to improve mental health, productive employment or income generation, quality of life and overall functioning among people with severe mental illness. ${ }^{9,10}$

The relative poverty and high levels of social and material difficulties in many urban areas in high-income countries have an adverse impact on mental well-being. ${ }^{11}$ In these communities the level of distrust and disengagement from mental health systems tends to be high. These are significant barriers to developing effective mental health systems and initiatives to enhance community resilience and well-being. Based on the experience in LMIC a development approach has the potential to improve community engagement and enhance the involvement of service users and their families in the design and delivery of mental healthcare. The development approach places an equal emphasis on enhancing livelihoods as in ensuring care and treatment and has the potential to improve health system capacity and social inclusion in high-income countries.

\section{Conclusion}

Innovations, such as task-sharing, are still at an early stage of development in LMIC. Although task-sharing has been found to be effective in diverse settings, it has not been scaled-up significantly in any country, nor been tested in any high-income countries, is restricted at present to a few mental health conditions and has not been evaluated for procedures such as diagnosis. ${ }^{6}$ The long-term sustainability of innovations such as task-sharing and community development is as yet unknown. There is a need to explore the potential effectiveness (and cost-effectiveness) of these innovations in high-income countries before they are implemented. We acknowledge that care systems cannot be simply copied from low- and middle-income to high-income countries any more than in the opposite direction. The very different political, cultural and economic contexts in these settings will have a bearing on the adaptability and success of such programmes. This means that differences in community organisation, culture and social contexts will need to be taken into consideration when transferring models of care from one setting to another. However, 
we believe that there is a strong case for adapting, testing and potentially implementing mental health innovations that have been proved to be effective and acceptable in resource-poor countries, in resource-rich countries. Given the seemingly perpetual crisis in relation to current services in high-income countries, there is a need to re-imagine and reshape mental healthcare in these countries. Learning from global mental health will be an important step in this direction.

It has been argued previously ${ }^{11}$ that wealthy countries can learn from prevention and management of mental health problems in low-income countries and this may help to address the remoteness of psychiatry and its allied professions from the communities they serve in many Western countries. ${ }^{6}$ Furthermore, this process of 'reverse transfer' may also prompt a move towards replacing the current dominance of psychiatric diagnostic categories/labels in favour of distress models that are more familiar and less stigmatising to those who use mental health services and their families. Delivery of care through collaborative models of care, as has been proposed in many LMIC settings, is also likely to ensure that the patient/family is at the centre and involve a partnership between the community-based worker and medical practitioners.

S. P. Sashidharan, MRCPsych, PhD, Ross White, PhD, Institute of Health and Wellbeing, University of Glasgow, Glasgow, UK; Roberto Mezzina, MD, Dipartimento di Salute Mentale, WHO collaborating Centre for Research and Training, Trieste, Italy; Stefan Jansen, PhD, Darius Gishoma, PhD College of Medicine and Health Sciences, Center for Mental Health, University of Rwanda, Rwanda

Correspondence: S. P. Sashidharan, Institute of Health \& Wellbeing, University of Glasgow, Admin BIdg, Mental Health and Wellbeing, Gartnavel Royal Hospital Glasgow G12 0XH, UK. Email: s.p.sashidharan@gmail.com

First received 5 Dec 2015, final revision 4 Mar 2016, accepted 7 Mar 2016

\section{References}

1 Drake RE, Binagwaho A, Martell HC, Mulley AG. Mental healthcare in low and middle income countries. BMJ 2014; 349: g7086.

2 Demyttenaere K, Bruffaerts R, Posada-Villa G, Gasquet I, Kovess V, Lepine JP, et al. Prevalence, severity, and unmet need for treatment of mental disorders in the World Health Organization World Mental Health Surveys. JAMA 2004; 291: 2581-90.

3 The King's Fund. Briefing: Mental Health Under Pressure. King's Fund, 2015 (http://www.kingsfund.org.uk/publications/mental-health-under-pressure).

4 Royal College of Psychiatrists. Mental Health and Social Inclusion. Royal College of Psychiatrists, 2009.

5 Patel V, Saxena S, De Silva M, Samele C. Transforming Lives, Enhancing Communities: Innovations in Mental Health. World Innovation Summit for Health. Qatar Foundation, 2013 (http://www.wish-qatar.org/summit/ inaugural-summit/reports/forum-reports).

6 Patel V. SUNDAR: mental health for all by all. BJPsych Int 2015; 12: 21-3.

7 van Ginneken N, Tharyan P, Lewin S, Rao GN, Meera SM, Pian J, et al. Non-specialist health worker interventions for the care of mental, neurological and substance-abuse disorders in low and middle-income countries. Cochrane Database Syst Rev 2013; 11: CD00914.

8 Imison C, Bohmer R. NHS and Social Care Workforce: Meeting our Needs Now and in the Future. The King's Fund, 2013.

9 Lund C, Waruguru M, Kingori K, Kippen-Wood S, Breuer E, Mannarath S, et al. Outcomes of the mental health and development model in rural Kenya: a 2-year prospective cohort intervention study. Int Health 2013; 5: 43-50.

10 Raja S, Underhill C, Shrestha P, Sunder U, Mannarath S, Wood SK, et al. Integrating mental health and development: a case study of the basic needs model in Nepal. PLoS Med 2012; 9: e1001261.

11 McKenzie K, Patel V, Araya R. Learning from low-income countries: mental health. BMJ 2004; 329: 1138-40. 